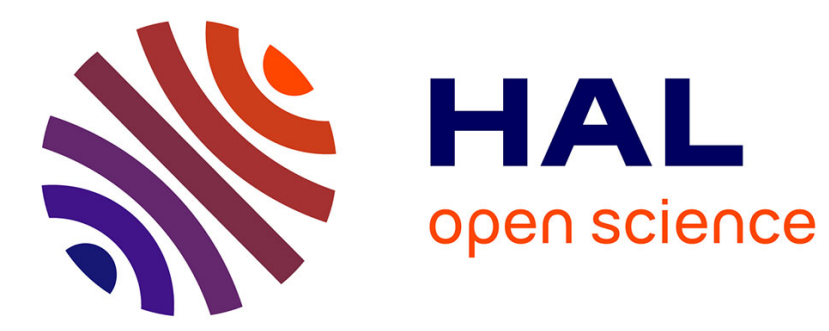

\title{
Cocaine increases dopaminergic connectivity in the nucleus accumbens
}

\author{
Jocelyne Caboche, Marc Dos Santos, Emma N Cahill, Gregory Dal Bo, Peter \\ Vanhoutte, Bruno Giros, Nicolas Heck, Marc dos Santos, Gregory Dal Bo
}

\section{- To cite this version:}

Jocelyne Caboche, Marc Dos Santos, Emma N Cahill, Gregory Dal Bo, Peter Vanhoutte, et al.. Cocaine increases dopaminergic connectivity in the nucleus accumbens. Brain Structure and Function, 2018, 223 (2), pp.913-923. 10.1007/s00429-017-1532-x . hal-03365853

\section{HAL Id: hal-03365853 \\ https://hal.science/hal-03365853}

Submitted on 5 Oct 2021

HAL is a multi-disciplinary open access archive for the deposit and dissemination of scientific research documents, whether they are published or not. The documents may come from teaching and research institutions in France or abroad, or from public or private research centers.
L'archive ouverte pluridisciplinaire HAL, est destinée au dépôt et à la diffusion de documents scientifiques de niveau recherche, publiés ou non, émanant des établissements d'enseignement et de recherche français ou étrangers, des laboratoires publics ou privés. 


\title{
Cocaine increases dopaminergic connectivity in the nucleus accumbens
}

\author{
Marc Dos Santos $^{1} \cdot$ Emma N. Cahill ${ }^{1,2} \cdot$ Gregory Dal Bo $^{3,4} \cdot$ Peter Vanhoutte $^{1} \cdot$ \\ Jocelyne Caboche ${ }^{1} \cdot$ Bruno Giros $^{1,3} \cdot$ Nicolas Heck $^{1}$
}

Received: 30 May 2017/ Accepted: 27 September 2017

(c) Springer-Verlag GmbH Germany 2017

\begin{abstract}
The development of addictive behavior is associated with functional and structural plasticity in the mesocorticolimbic pathway. Increased connectivity upon cocaine administration has been inferred from increases in dendritic spine density, but without observations of presynaptic elements. Recently, we established a method that enables analyses of both dendritic spines and glutamatergic boutons and presented evidence that cocaine induces changes in striatal connectivity. As the pharmacological and behavioral effects of cocaine directly implicate dopaminergic neurons and their afferents, a remaining question is whether dopaminergic striatal innervations also undergo structural plasticity. To address this issue, we generated transgenic mice in which the fluorophore tdTomato is expressed under the promoter of the dopamine transporter gene. In these mice, specific labeling of dopaminergic boutons was observed in the striatum. Of note, the accordance of our results for control mice with previous electron microscopy studies confirms that our method can be used to decipher the spatial organization of boutons in relation to dendritic elements. Following
\end{abstract}

Nicolas Heck

nicolas.heck@upmc.fr

1 Sorbonne Université, UPMC Univ Paris 06, INSERM, CNRS, Neurosciences Paris Seine, Institut de Biologie Paris Seine (NPS, IBPS), 75005 Paris, France

2 Present Address: Department of Psychology, Behavioral and Clinical Neuroscience Institute, University of Cambridge, Cambridge, UK

3 Department of Psychiatry, Douglas Mental Health Research Center, McGill University, Montreal, QC, Canada

4 Present Address: Département de Toxicologie et risque chimiques, IRBA, Brétigny sur Orge, France repeated cocaine administration that led to behavioral locomotor sensitization, an increased density of dopaminergic boutons was observed 1 day later in the nucleus accumbens shell specifically, and not in other striatal regions. Combined labeling of dopaminergic boutons and striatal dendrites showed that cocaine significantly increased the percentage of dendritic spines associated with a dopaminergic bouton. Our results show that chronic cocaine administration induces structural plasticity of dopaminergic boutons that could participate in dopaminedependent neuronal adaptations in the striatum.

Keywords Dopamine $\cdot$ Cocaine $\cdot$ Dendritic spine $\cdot 3 D$ imaging $\cdot$ Striatum

\section{Introduction}

It is now widely accepted that structural plasticity is implicated in long-term adaptations that underlie several psychiatric disorders, including drug addiction (Bernardinelli et al. 2014; Russo et al. 2010). Structural plasticity is, however, most often studied at the level of the postsynaptic structure only. Although observation of spine density along the dendrite is a valuable estimation of connectivity, the knowledge of the structural plasticity at the level of the axon is a critical issue for a proper understanding of synapse formation (Wierenga et al. 2008; Hübener and Bonhoeffer 2010; Schoonover et al. 2014; Yang et al. 2016; Johnson et al. 2016). Recently, we developed a method for observing and analyzing glutamatergic pre and postsynapses (Heck et al. 2015). This approach enabled us to demonstrate that synapse formation indeed occurred after cocaine administration, for which an increase in spine density had previously been shown 
(Russo et al. 2010). Those spines also receive dopaminergic inputs from the mesencephalon, which encode rewardprediction error signals (Schulz 2002). By blocking the dopamine transporter (DAT), cocaine induces an increase in extracellular dopamine (DA) levels (Di Chiara and Imperato 1988) and thereby hijacks, the reward-driven learning circuit. This process is thought to promote a pathological form of memory leading to the addicted state (Hyman et al. 2006). The associated mechanisms include synaptic plasticity, activation of signaling pathways, gene expression changes and epigenetic modifications in striatal projection neurons (SPN), which all contribute to the longterm modifications of the network that sustains behavioral alterations induced by drugs of abuse (Lüscher and Malenka 2011; Robison and Nestler 2011). Whether structural changes occur at the level of DA afferents onto SPN remains an open question. Among others SPN receive glutamatergic as well as DA inputs, which synergistically activate the Extracellular-signal Regulated (ERK) pathway (Pascoli et al. 2014). Hence, a change in DA synaptic organization onto SPN could participate in the cellular events involved in cocaine-induced neuroplasticity. DA neurons located in the midbrain, project axons that spread their arborization in the striatum (Matsuda et al. 2009; Aransay et al. 2015; Rodríguez-López et al. 2017). The released DA modulates the activity and plasticity of striatal neurons (Gerfen and Surmeier 2011; Tritsch and Sabatini 2012). DA boutons can form synaptic contacts on the neck of dendritic spines of SPN, but most boutons do not establish any synaptic contact (Descarries et al. 1996; Sesack and Grace 2010; Bérubé-Carrière et al. 2012). DA afferents thus form a dense lattice, and the released DA acts through volume transmission within the striatum (Moss and Bolam 2008). Here, the density and spatial organization of DA boutons was measured in the striatum of mice that showed locomotor sensitization following repeated administration of cocaine. The present study reveals a structural plasticity of the DA innervation on the nucleus accumbens shell, which likely participates in the neuronal and behavioral adaptations induced by cocaine.

\section{Materials and methods}

\section{Mice}

Animal care was conducted in accordance with the European Commission Guidelines on the Care and Use of Laboratory Animals (Directive 86/609/EEC), and the experiments were approved by the local ethic committee C2EA-05. Mice expressing the Cre recombinase under the DAT promoter (Turiault et al. 2007) were crossed with mice carrying a ROSA-tdTomato reporter acquired from
Jackson Laboratories [Gt(ROSA)26Sortm9(CAG-tdTomato)Hze] (Madisen et al. 2010). Male mice from the C57BL6 strain weighing 22-24 g were housed five per cage and acclimatized to the laboratory conditions $(12 \mathrm{~h}$ light/dark cycle, $21 \pm 1{ }^{\circ} \mathrm{C}$ room temperature) with ad libitum access to food and water.

\section{Locomotor sensitization}

Adult male mice were injected intraperitoneally with saline $(0.9 \% \mathrm{NaCl})$ and placed in the activity box for $60 \mathrm{~min}$ on three consecutive days for habituation. The mice were then treated with either saline or cocaine $(20 \mathrm{mg} / \mathrm{kg}$ in saline, Sigma-Aldrich) for five consecutive days and perfused on the sixth day for morphological analysis. Locomotor activity was measured for $30 \mathrm{~min}$ after the saline or cocaine injection using rectangular activity boxes (Immetronic). Locomotor activity was counted as total travel distance, measured by interruption of photocell beams located $15 \mathrm{~mm}$ above the floor.

\section{Slice preparation}

Mice were deeply anesthetized with an intraperitoneal injection of pentobarbital and perfused intracardially with $30 \mathrm{ml}$ of paraformaldehyde (PFA) at $1.5 \%$ in $0.1 \mathrm{M}$ phosphate buffer, $\mathrm{pH} 7.4$ at $4{ }^{\circ} \mathrm{C}$. The brain was removed from the skull and placed in $1.5 \%$ PFA for $1 \mathrm{~h}$ at $4{ }^{\circ} \mathrm{C}$. Slices of $150 \mu \mathrm{m}$ thickness were cut in the frontal plane using a vibratome (Leica) and stored in phosphate-buffered saline (PBS).

\section{Immunofluorescence}

Brain slices were permeabilized for 30 min with $0.1 \%$ Triton X-100 and 3\% bovine serum albumin (BSA) in PBS. Rabbit polyclonal antibodies against tyrosine hydroxylase (TH) (1/1000, Sigma) or vesicular monoamine transporter2 (VMAT2) (1/1000, C3Bf from B. Giros Lab) were incubated overnight at $4{ }^{\circ} \mathrm{C}$ in $0.01 \%$ Triton and 3\% BSA in PBS. The slices were washed in PBS and incubated with the secondary antibody coupled to Alexa 488 (anti-rabbit 1/500, Invitrogen) for $2 \mathrm{~h}$ in $0.01 \%$ Triton 3\% BSA in PBS. The slices were washed in PBS and mounted in Prolong (Invitrogen).

\section{Image acquisition}

The principles of image acquisition and deconvolution have been extensively described in Heck et al. (2012). Image stacks coded in 16-bit were taken using a confocal laser scanning microscope (SP5, Leica) equipped with a 1.4 NA objective (oil immersion, Leica) with a pinhole 
aperture set to 1 Airy unit, pixel size of $60 \mathrm{~nm}$ and $\mathrm{z}$-step of $200 \mathrm{~nm}$. The excitation wavelength and emission range were 488 and 500-550 for Alexa 488 and DiO, 561 and 570-650 for tdTomato. Laser intensity and PMT gain were set so that each image occupied the full dynamic range of the detector. Metrology measurements were regularly performed using fluorescent beads to confirm proper laser alignment, laser power and field homogeneity using the ImageJ-based MetroloJ plugin. Deconvolution with experimental point spread function from fluorescent beads using a maximum likelihood estimation algorithm was performed with Huygens software (Scientific Volume Imaging). One hundred fifty iterations were applied in classical mode, background intensity was averaged from the voxels with lowest intensity, and signal to noise ratio values were set to a value of 15 for Tomato signal and 20 for $\mathrm{DiO}$ dendritic signal.

\section{Segmentation of synaptic objects, volume measurement, co-localization analysis}

Three-dimensional segmentation and analysis of tdTomatopositive and immunolabeled boutons were performed using the procedure described in Heck et al. (2015). The procedures were run using custom ImageJ macros and the $3 \mathrm{D}$ functionalities of the 3D ImageJ Suite plugin. Of note, the workflow has been implemented in a user-friendly plugin called DiAna (Gilles et al. 2017). For image segmentation, a 3D watershed procedure defined the boundaries of space regions that contained objects, and local maxima were detected in the image. Histogram analysis and image inspection allowed a threshold intensity to be defined so that only local maxima from objects were retained. For each local maximum, corresponding to one object, the $3 \mathrm{D}$ intensity distribution centered on the local maxima was computed and fitted to a Gaussian curve. The intensity value covering $95.4 \%$ of the surface of the Gaussian curve was determined as the intensity threshold of the border of the object. Voxels located around the local maximum were then included in the object if they fulfilled the following three criteria of acceptance: intensity higher than the threshold; intensity lower than the previously included voxel; and inclusion of neighboring voxels; the last criteria was defined for acute object shape extraction). As the segmentation of each object is based on the analysis of its $3 \mathrm{D}$ intensity distribution, the procedure ensures that boutons of similar size but different intensity are extracted as objects of similar size. The volume of each extracted object was computed using the 3D ImageJ Suite plugin.

Object-based co-localization analysis was performed following segmentation. For each region of interest corresponding to a segmented tdTomato-positive DA bouton, the intensity in the other segmented channel was measured. Hence, co-localization was considered to occur when a TH or VMAT2 segmented object overlapped with a tdTomato-positive segmented object. For each pair of colocalizing objects, the 3D center-to-center distance between the two objects was computed using the 3D ImageJ Suite plugin.

\section{Dendrite labeling and dendritic spine analysis}

Dendrites and dendritic spines were labeled using the Diolistic technique described by Gan et al. (2000). Briefly, $50 \mathrm{mg}$ of tungsten beads (Bio-Rad) was mixed with $3 \mathrm{mg}$ of solid green $\mathrm{DiO}$ (3,3'-dioctadecyloxacarbocyanine perchlorate, Molecular Probes) dissolved in methylene chloride. DiI-coated beads were coated on the inner surface of a polyvinylpyrrolidone (Sigma-Aldrich)-pretreated Teflon tube. The tube was then cut in pieces, which were inserted as cartridges in a gene gun device (Bio-Rad). Helium gas pressure (150 psi) applied through the gene gun ejected the beads out of the cartridge onto the brain slice. The beads were delivered through a $3-\mu \mathrm{m}$ pore-size filter (Isopore polycarbonate, Millipore) to avoid clusters. After labeling, the slices were kept in PBS at room temperature for at least $2 \mathrm{~h}$ and mounted in Prolong Gold.

Dendritic spine density was defined as the number of spines normalized to a $10 \mu \mathrm{m}$ length of dendrite. Neuronstudio software (version 0.9.92) (Rodriguez et al. 2008) was used to reconstruct the dendrite and detect dendritic spines. When necessary, manual correction was applied. For each striatal neuron, a dendritic segment of 20-70 $\mu \mathrm{m}$ in length and distant from at least $50 \mu \mathrm{m}$ from the soma or after the first branching point was considered. The percentage of spines for which the neck was in contact with a dopaminergic bouton was manually counted by visual inspection in 3D through confocal image stacks.

\section{Computation of 3D distance between dopaminergic boutons and potential receptor targets}

A custom ImageJ macro was built, incorporating functions from the 3D ImageJ Suite plugin. For each segmented image of DA boutons, 100 dots (one voxel size) were placed at random positions within the $3 \mathrm{D}$ space in between DA boutons. A mask was built so that the points were never placed within cell soma. The minimal distance from each point to the border of the closest DA bouton was measured. The distributions of minimal distances for the images from the saline and cocaine conditions were then computed. 


\section{Results}

\section{Observation and identification of dopaminergic boutons in the striatum}

To observe DA boutons in the striatum, a mouse line expressing the Cre recombinase under the control of the DA neuron-specific promoter of the DAT gene was crossed with a mouse line carrying a floxed gene driving the expression of the fluorescent protein tdTomato (Fig. 1a). Hence, DA neurons express tdTomato in these mice. The expression level and brightness of tdTomato fluorescence allowed for the observation of boutons in the striatum, whereas signals in the axons were not detected with the chosen image acquisition parameters. tdTomato-positive spots were segmented from 3D images (Fig. 1b). The average volume of the segmented objects was $0.297 \pm 0.017 \mu \mathrm{m}^{3}$, corresponding a sphere $0.827 \mu \mathrm{m}$ in diameter, a value consistent with the size of DA boutons reported in studies conducted in dorsal striatum with serial electron microscopy (Moss and Bolam 2008). Slightly smaller diameters have been reported in the ventral striatum in a 2D electron microscopy study (Bérubé-Carrière et al. 2012). It is noteworthy that optical microscopy has lower resolution in $z$-axis compared to $x y$ axis, and, despite restoration by deconvolution, our measurements of volumes may be over-estimated. This however would not impair the comparison of volumes objects between different conditions. The specificity of the labeling was confirmed by immunostaining against $\mathrm{TH}$, which is expressed in DA axons innervating the striatum (Fig. 1c). Following segmentation, 3D co-localization analysis revealed extensive overlap, with $88.4 \%$ of tdTomato-positive objects co-localizing with TH objects $(n=1883$ tdTomato-positive objects from $n=2$ mice). To confirm that the tdTomato-positive objects contained dopaminergic vesicles, immunostaining against VMAT2 was performed (Fig. 1d). Of all tdTomato-positive segmented objects, 95.2\% co-localized with VMAT2 segmented objects ( $n=4610$ tdTomato-positive objects from $n=2$ mice). The center-to-center distance from tdTomato-positive objects to VMAT2 objects was computed in 3D. Measurements from $n=3775$ object pairs retrieved a value of $227 \pm 0.008 \mathrm{~nm}$, which indicates accurate co-localization and thus confirms that the tdTomato-positive objects are DA boutons.

\section{Chronic cocaine exposure induces structural plasticity of dopaminergic boutons in the nucleus accumbens}

The possible reorganization of DA inputs upon repeated cocaine exposure was studied in a locomotor sensitization paradigm (Fig. 2a). Mice received either a single dose of
Fig. 1 Specific detection of dopaminergic boutons in the striatum. a To specifically label DA boutons in the mouse brain, the DAT::Cre mice were crossed with the tdTomato "flex" mice to specifically express the fluorescent protein tdTomato in DA neurons and in their striatal afferents. b Single plane from a confocal image stack showing tdTomato labeling. False-color rendering enables better visualization of the high heterogeneity in the size and fluorescence intensity of the objects. Following a custom segmentation procedure, tdTomatopositive objects are extracted. The output of the segmentation is shown on the same single plane of the confocal stack as the original image, and with $3 \mathrm{D}$ volume rendering. c Immunohistochemistry for TH shows co-localization with tdTomato-positive objects. d Immunohistochemistry for VMAT2 shows co-localization with tdTomatopositive objects. Scale bar in b $1 \mu \mathrm{m} ; \mathbf{c}, \mathbf{d}=2 \mu \mathrm{m}$. $S N$ substantia nigra, VTA ventral tegmental area, NAc nucleus accumbens

cocaine or saline daily, and their locomotor activity was recorded. The same dose of cocaine $(20 \mathrm{mg} / \mathrm{kg})$ induced a progressive increase in locomotor activity compared to saline treated mice. This increased behavioral output induced by cocaine reveals that the neuronal network undergoes changes, such that the next administration of the same dose of cocaine acts on a primed network. One day after the last cocaine injection, images of the DA boutons were acquired by confocal microscopy in the different regions of the striatum: the medial and lateral part of the dorsal striatum and the shell and core of the nucleus accumbens (NAc). The sections imaged were located $0.9-1.2 \mathrm{~mm}$ rostral from bregma. The images were deconvolved and segmented to measure bouton density and volumes (Fig. 2b). A significant increase in DA bouton density was measured in the NAc shell, but not in the other regions of the striatum (Fig. 2c). Three-dimensional morphometric analysis revealed a decrease in the volume of the boutons in both the shell and the core of the NAc, but not in the dorsal striatum (Fig. 2d).

Many DA boutons are devoid of synapse, so the released DA diffuses to bind with its' receptors (Descarries et al. 1996; Moss and Bolam 2008; Robinson et al. 2008). DA receptors are found on the dendritic shaft and dendritic spines of SPN as well as on glutamatergic and dopaminergic boutons (Sesack et al. 1994; Yung et al. 1995; De Mei et al. 2009; Uchigashima et al. 2016). Therefore, a change in the density and volume of DA boutons could modify the range of DA volume transmission and access to DA receptors. To address this hypothesis, a model was built whereby single points, representing putative receptor sites, were inserted at random positions in images of DA boutons obtained from saline and cocaine treated mice. The minimal distance between each point and the borders of DA boutons was measured in the three dimensions (Fig. 2e). In images from saline treated mice, $85.9 \%$ of DA boutons were less than $1 \mu \mathrm{m}$ away from the nearest putative target (Fig. 2f). The cumulative frequency distribution of the 
A

DAT :: Cre mice

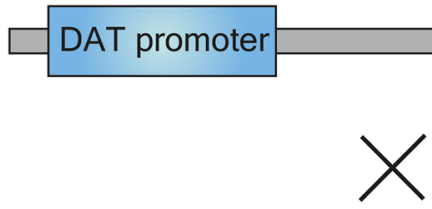

«Flex» tdTomato mice

\section{CRE}

- CAG promoter $<-$ STOP $\leq-$ tdTomato

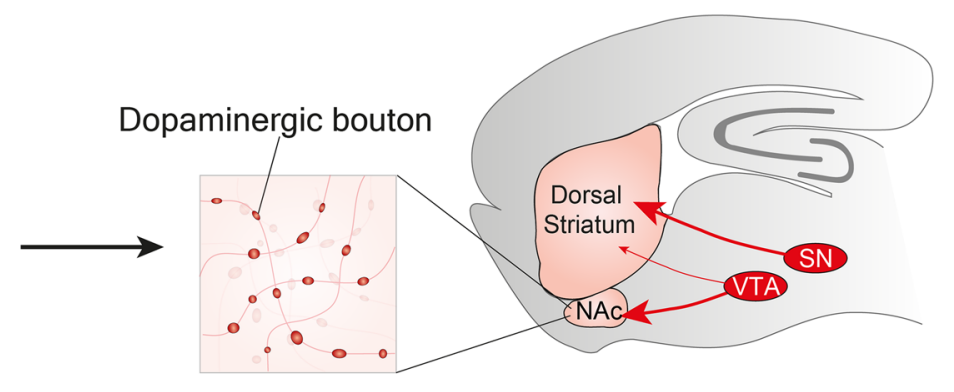

B

tdTomato spots

Adaptive segmentation
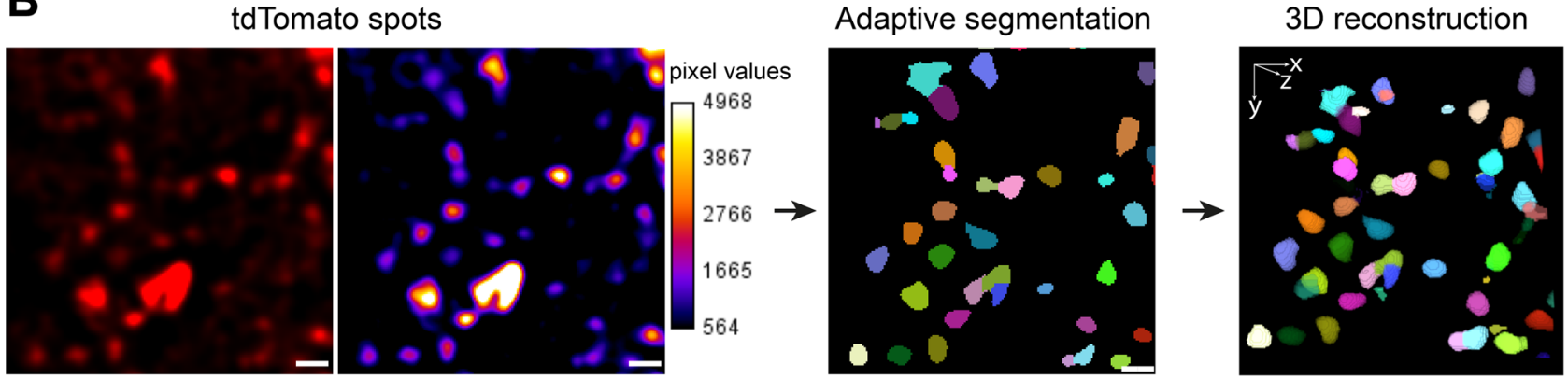

C $\mathrm{TH}$

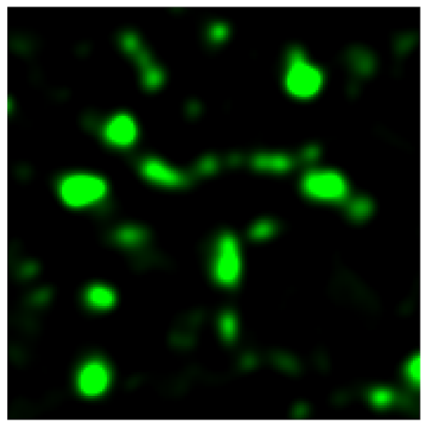

D VMAT2

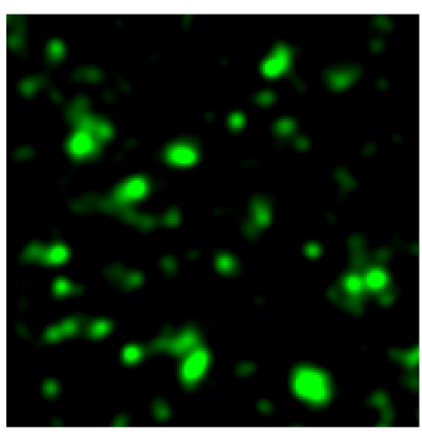

tdTomato

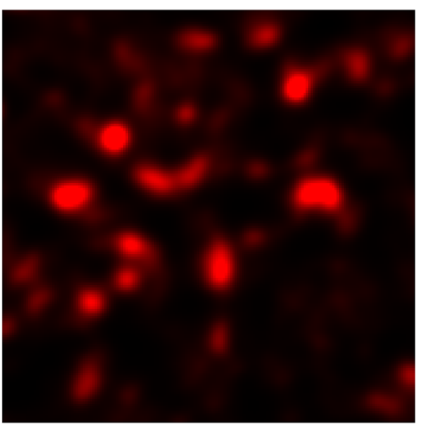

tdTomato

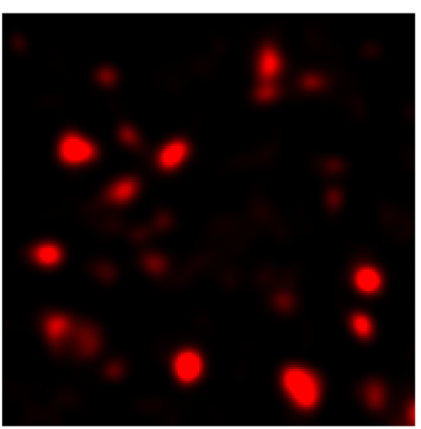

Merge

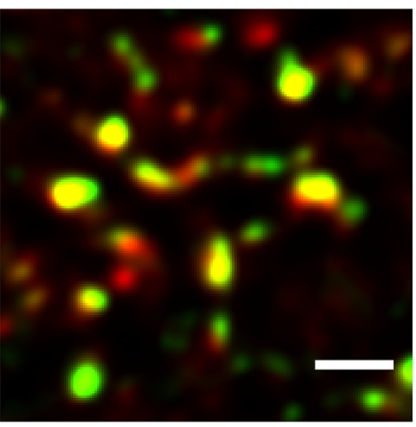

Merge

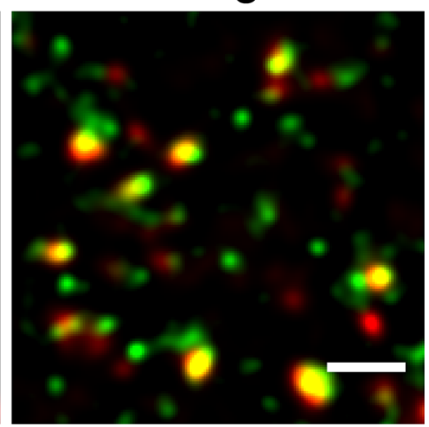

3D Merge

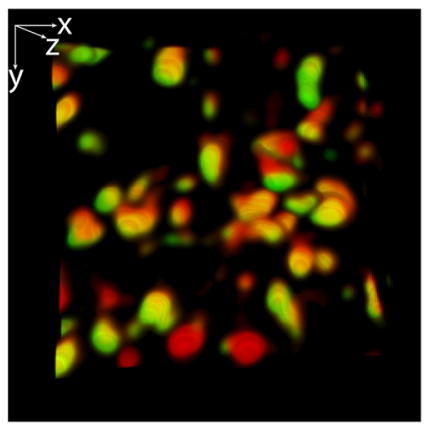

3D Merge

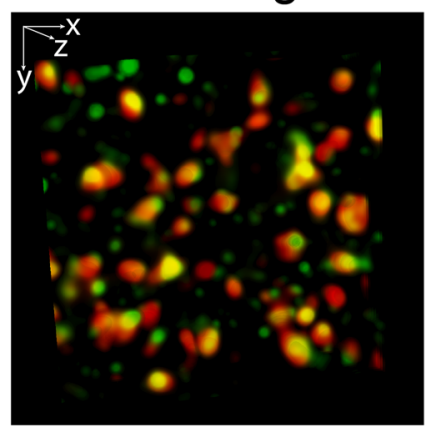


minimal distances from the borders of DA boutons to putative DA receptors showed that in images from mice that received repeated cocaine administrations, the minimal distances were shorter than in the saline condition, with $89.8 \%$ of DA boutons located less than $1 \mu \mathrm{m}$ away from the nearest putative target in the cocaine condition (Fig. 2f).

Taken together, these results reveal that repeated administration of cocaine induces structural plasticity of the DA afferents in the ventral part of the striatum, a cerebral structure strongly implicated in the early phase of cocaine addiction (Steketee and Kalivas 2011).

\section{Spatial relationship between dopaminergic boutons and dendritic spines of striatal projection neurons}

Dopamine boutons in the striatum are not necessarily associated with a postsynaptic structure but can contact the dendritic spines of SPN (Sesack and Grace 2010; Moss and Bolam 2008; Xu et al. 2012; Uchigashima et al. 2016). Because repeated exposure to cocaine induces an increase in dendritic spine density (Russo et al. 2010; Heck et al. 2015) and our results show an increase in DA bouton density in the NAc shell, it raises the intriguing question as to whether combined pre and post-synaptic structural plasticity could modify the spatial relationship between the dendritic spines and the DA boutons. To address this question, SPN were sparsely labeled with a green membrane-associated fluorochrome in slices of saline or cocaine treated transgenic mice, allowing the simultaneous observation of dendritic spines and DA boutons (Fig. 3a, b). Following repeated cocaine administration, which induced locomotor sensitization (see Fig. 2a), a significant increase in dendritic spine density was measured in the NAc shell compared to saline control conditions (Fig. 3c). The occurrence of contacts between DA boutons and the neck part of the dendritic spines was then monitored. In saline conditions, $22.8 \pm 1.5 \%$ of dendritic spines were in direct contact with a DA bouton (Fig. 3d, $n=27$ dendrites with 1257 spines). In mice treated with cocaine, the percentage of spines in contact with a DA bouton was $35.6 \pm 0.7 \%$, corresponding to an increase of $56 \%$ in comparison to saline controls (Fig. 3d, $n=21$ dendrites with 783 spines). Altogether, these results highlight that cocaine induces a spatial rearrangement of the DA connectivity to favor putative synaptic contacts.

\section{Discussion}

Cocaine, by inhibiting the uptake of released DA, induces an elevated level of extracellular DA that triggers adaptations in striatal neurons leading to addictive behavior.
Whereas dendritic spine density (Russo et al. 2010) and glutamatergic connectivity (Heck et al. 2015) changes induced by cocaine have been extensively studied, a possible structural reorganization of dopaminergic afferents remained to be addressed. In the present study, we used the DAT-tdTomato transgenic mouse line that allowed the direct observation of DA boutons in the striatum. Following repeated cocaine administrations that induced locomotor sensitization, an increase in DA bouton density was observed in the NAc shell as well as an increase in the percentage of dendritic spines in contact with a DA bouton.

Our methodological approach enabled the examination of the 3D organization of DA boutons within the striatum. It is important to note that our measurements in control conditions are in accordance with results from a previous electron microscopy study (Moss and Bolam 2008; BérubéCarrière et al. 2012). Analysis of serial electron microscopy images enabled the percentage of dendritic spines in contact with a DA bouton to be estimated at $20 \%$; and measurements of distances between randomly selected points and DA presynapses revealed that $96 \%$ of randomly selected points were located less than $1.04 \mu \mathrm{m}$ from DA boutons (Moss and Bolam 2008). The spatial relationship between DA boutons and dendritic spines from striatal neurons we described, as well as the distance between DA boutons and potential receptor sites we measured, fit remarkably well with the conclusion of the study of Moss and Bolam, although it should be noted that this study was conducted in the dorsal striatum. Hence, our method, associating optical confocal microscopy with image deconvolution and 3D analysis, is an efficient strategy for the investigation of spatial organization of boutons, with the advantage of sampling large volumes of brain tissue and providing information from thousands of objects. Accordingly, recently published studies, including from our laboratory (Heck et al. 2015; Schoonover et al. 2014; Yang et al. 2016), demonstrated that optical microscopy combined with careful analysis can successfully assess the spatial organization of boutons and dendritic spines.

The possible changes of DA innervation following cocaine administration have been previously addressed indirectly using western blotting and immunohistochemistry against $\mathrm{TH}$, an enzyme involved in DA synthesis. However, those approaches either did not detect an increase in axonal TH expression (Beitner-Johnson and Nestler 1991; Sorg et al. 1993) or led to contradictory results (Todtenkopf and Stellar 2000; Todtenkopf et al. 2000; Lee et al. 2011). Those methods likely suffered from false negatives artefacts inherent to immunostaining techniques, undersampling due to two-dimensional analysis and false positives due to the presence of noradrenergic fibers (Sesack and Grace 2010) and a subpopulation of THpositive striatal interneurons (Ibáñez-Sandoval et al. 2010). 
A
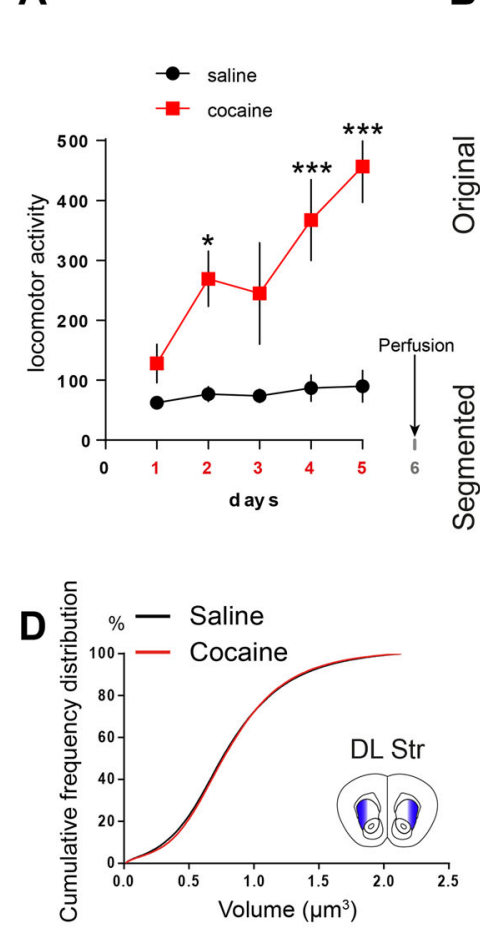

E

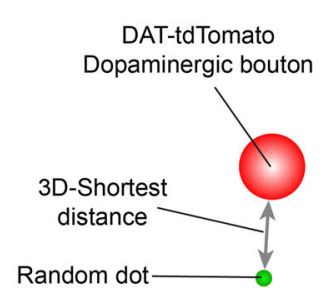

B

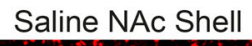

\section{C saline cocaine}

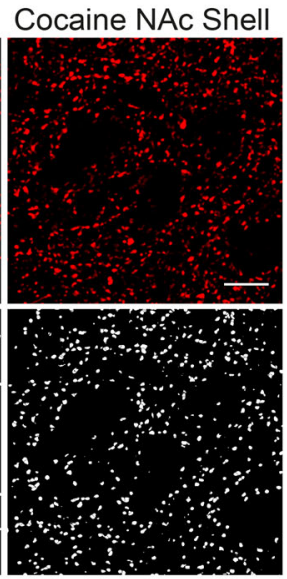

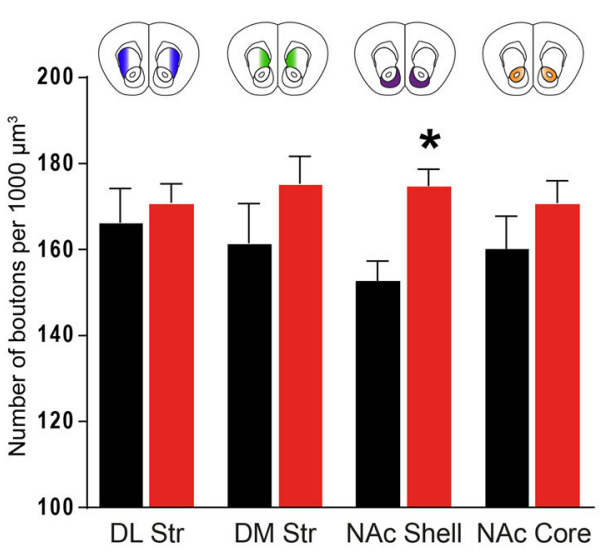
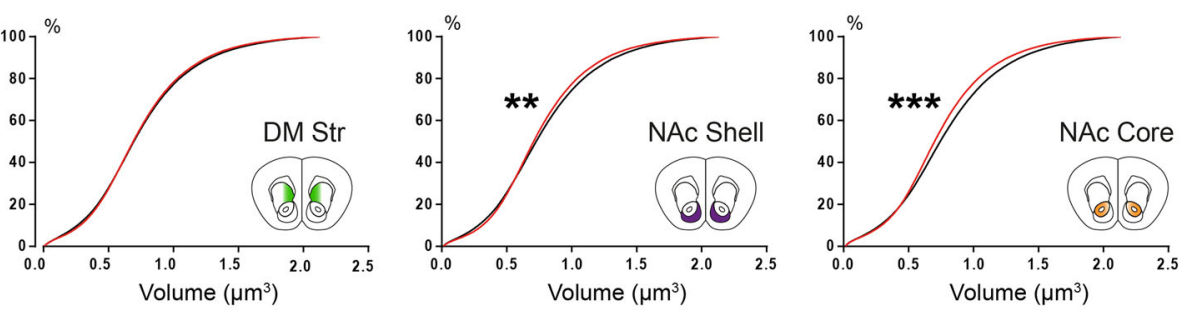

$\mathbf{F}$
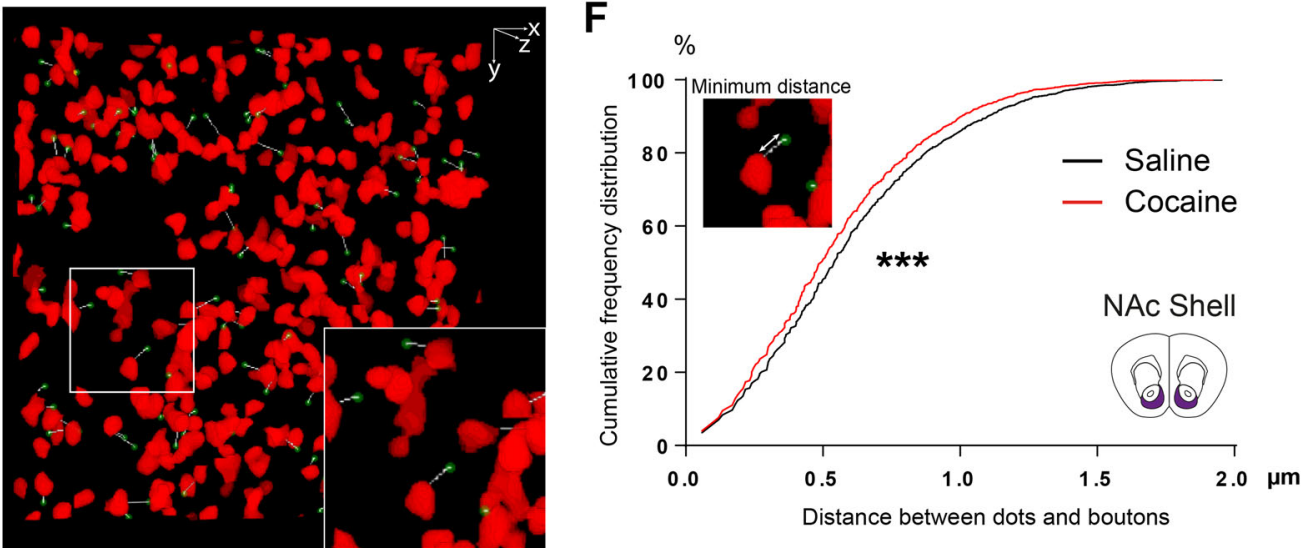

Fig. 2 Repeated cocaine exposure increases dopaminergic innervation in the nucleus accumbens shell. a Transgenic mice injected daily with cocaine (ip. $20 \mathrm{mg} / \mathrm{kg}$ ) present locomotor sensitization, defined as a progressive increase in locomotor activity upon repeated injection of the same dose of cocaine. Two-way ANOVA, Bonferroni post hoc test: cocaine day 1 vs. day 5: $p<0.001$, saline day 1 vs. day 5: $p>0.05$; saline day 5 vs. cocaine day 5: $p<0.001$. ${ }^{* * *} p<0.001$. b DA boutons were imaged and segmented in $3 \mathrm{D}$, as illustrated by a single plane from confocal stacks imaged in the NAc shell. c The measurement of DA bouton density reveals an increase in the NAc shell but not in the other striatal regions. One-way ANOVA, Dunn's multiple comparison test, Saline vs. cocaine: NAc Shell: $152.7 \pm 4.6$ vs. $174.8 \pm 3.9, p<0.05$, NAc Core: $160 \pm 7.6$ vs. $170 \pm 5.2$, DM Str: $161 \pm 9.2$ vs. $175 \pm 6.4$, DL Str: $166 \pm 8$ vs. $170 \pm 4.4$. $n=6-7$ mice per condition. $* p<0.05$. d Cumulative distributions of the volumes of DA boutons analyzed in the different striatal regions. Bouton size is decreased only in the ventral parts of the striatum. Kolmogorov-Smirnov test. $n=114,265-147,076$ boutons from $n=6-7$ mice per condition. e Single dots are inserted at random locations in segmented confocal stacks imaged in the NAc shell from transgenic mice treated with saline or cocaine. The 3D minimal distance from each dot to the borders of DA boutons is computed. Three-dimensional volume rendering showing the dots (green), the segmented DA boutons (red) and the minimal distance computed (white). The single dots have been enlarged in this illustrative example for better visualization. f Cumulative frequency distribution of minimal distance between single dots placed randomly and the border of the closest DA bouton in the shell of the NAc. 100 dots were placed in 22 (saline condition) and 26 (cocaine condition) confocal image stacks each containing an average number of 5428 DA boutons. In cocaine-treated mice, the minimal distance from DA boutons to randomly located dots is reduced compared to salinetreated mice. Kolmogorov-Smirnov test, $p<0.001$. Scale bar in b $10 \mu \mathrm{m}$. DM Str dorsomedial striatum, $D L$ Str dorsolateral striatum, $N A c$ nucleus accumbens 


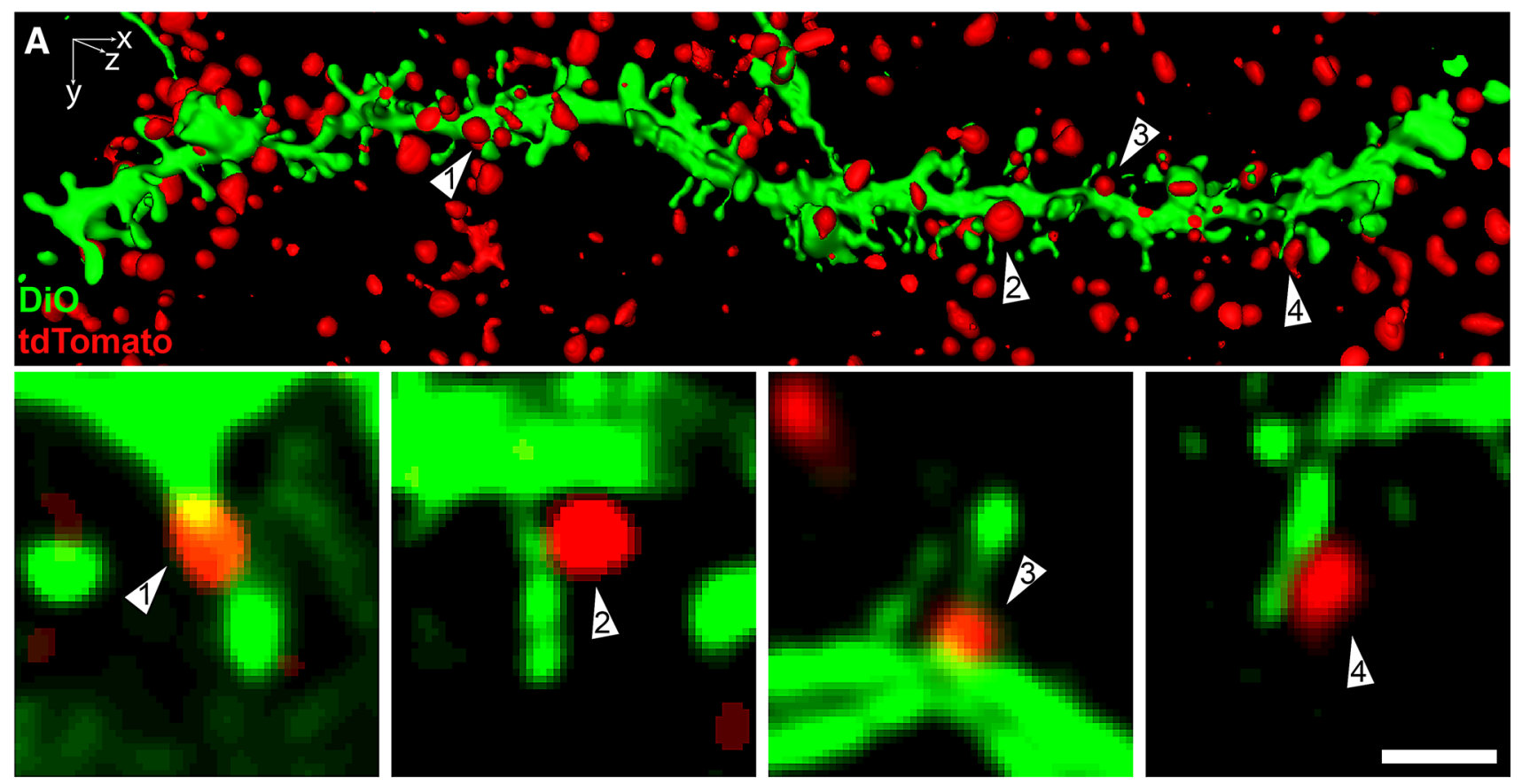

\section{B}
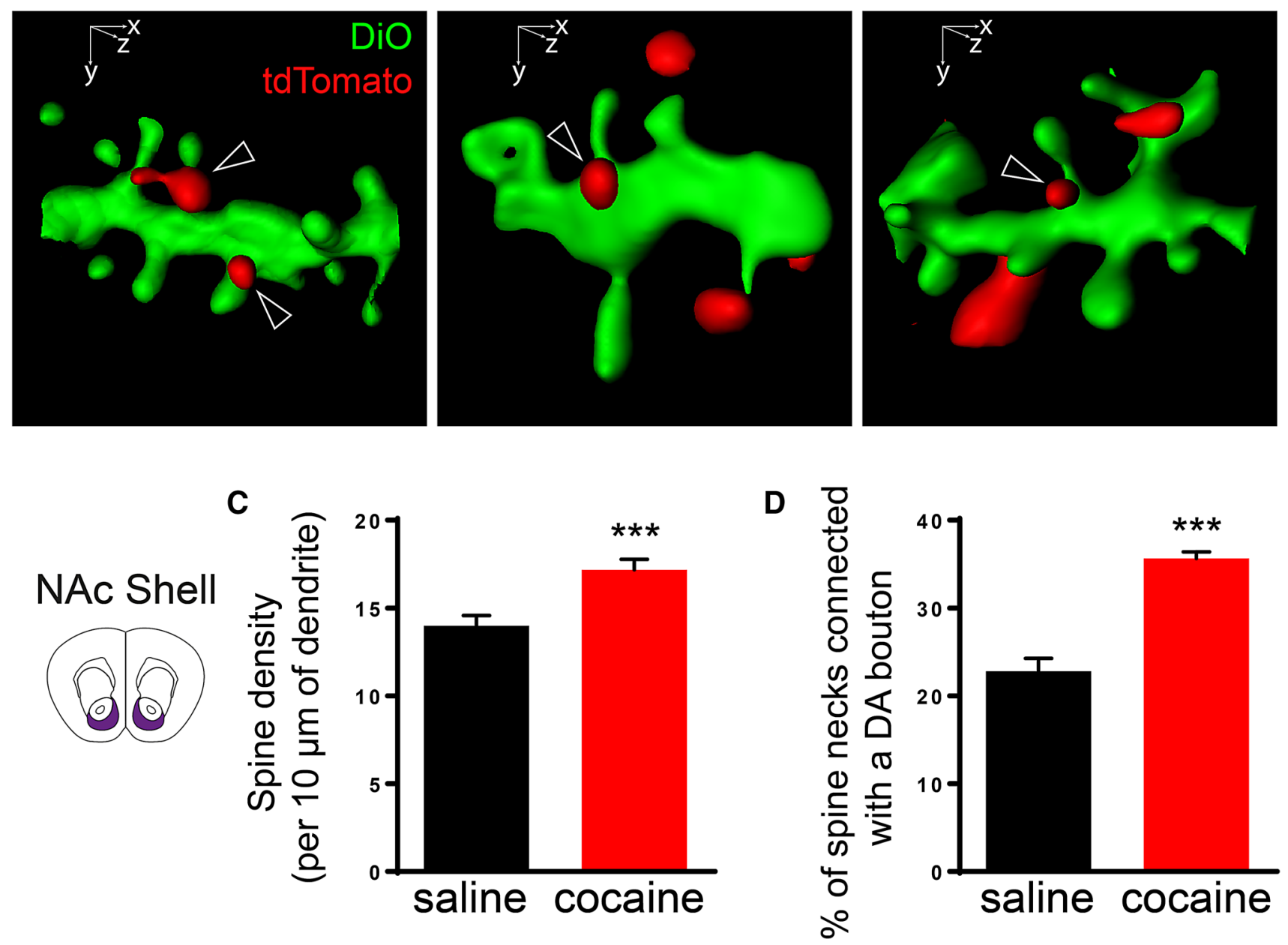
4Fig. 3 Repeated cocaine exposure increases dopaminergic connectivity in the nucleus accumbens. Contacts between DA boutons (red) and the neck of dendritic spines labeled with $\mathrm{DiO}$ (green) were detected in the NAc shell. a Upper panel: Three-dimensional surface rendering of a confocal microscopy image stack showing the dendrite and dendritic spines and the DA boutons. Lower panel: close-ups showing single planes from the same image stack. b 3D surface rendering of contacts between DA boutons and the neck of dendritic spines. c Spine density on SPN is increased in cocaine-treated mice compared to saline condition. Spine density was measured in the NAc shell of transgenic mice. $\mathbf{d}$ The percentage of DA boutons in contact with the neck of a dendritic spine is increased in cocaine-treated mice compared to saline condition. $t$ test in $\mathbf{c}$ and $\mathbf{d}: p<0.001, n=27$ and 21 dendrites with 1257 and 783 spines in saline and cocaine condition, respectively. ${ }^{* * *} p<0.001$. Scale bar in a $1 \mu \mathrm{m}$

Here, we clearly demonstrate that $24 \mathrm{~h}$ following chronic administration of cocaine, the density of DA boutons is increased. One possible consequence could be an enhancement of DA transmission. The extracellular level of DA has been measured by microdialysis and voltammetry techniques in the NAc shell in a cocaine administration protocol similar to the one applied in our study. During the early phase of abstinence, the basal level of DA was found to be either unchanged (Addy et al. 2010) or decreased (Chefer and Shippenberg 2002) in sensitized animals, whereas a challenge of cocaine increased DA levels to a greater magnitude in sensitized compared to saline treated animals (Addy et al. 2010; Chefer and Shippenberg 2002). Our observation of an increase in DA terminal density seems in contradiction with the decreased or unchanged basal DA levels may seem paradoxical. One possible explanation would be that not all DA boutons are functional, as it has been shown that some boutons containing vesicle clusters do not release DA (Pereira et al. 2016). In this respect, the decrease in DA boutons volume observed in the NAc might indicate alterations in DA storage. On the other hand, the increase in DA terminal density fits with the increased DA levels upon cocaine challenge in sensitized animals. Several mechanisms could explain the changes in extracellular DA levels in the striatum of sensitized rodents, including changes of DA neuron activity, probability of DA release, regulation of DA release through DA presynaptic receptors or DAT expression level (Kuhar and Pilotte 1996; Robinson et al. 2008; Kalivas and Stewart 1991). Here, we show that chronic administration of cocaine induces an increase in the number and volume of DA axonal boutons. Hence, this parameter should be taken into consideration for the global understanding of the consequences of cocaine administration on DA homeostasis in the striatum. Furthermore, it would be of interest to assess whether the changes we describe are dynamic through withdrawal period and upon cocaine challenge.
Interestingly, the increase in DA bouton density was restricted to the shell part of the NAc. Without precluding involvement of other brain structures and circuit-level mechanisms, the shell is critically involved in cocaine sensitization (Steketee and Kalivas 2011), with chronic cocaine administration inducing synaptic plasticity (Thomas et al. 2001) and signaling pathway activation (Robison et al. 2013) in the shell, but not in the core of the NAc. Different DA neurons project to sub-divisions of the NAc (Lammel et al. 2014) and it has been shown that acute cocaine administration elicits increase in DA release in the shell but not in the core (Aragona et al. 2008), evokes synaptic plasticity in DA neurons projecting to the shell but not to dorsal striatum (Lammel et al. 2011), and induces increase or decrease of the bursting activity in different neurons from the ventral tegmental area (Mejias-Aponte et al. 2015). Those results provide experimental evidence that cocaine can differentially affect dopaminergic neurons projecting to different brain areas, forming a basis on which further experiments could be conducted to determine the mechanisms by which cocaine induce an increase in bouton density specifically in the shell.

The observation of DA boutons devoid of synaptic membrane specialization has led to the idea of volume transmission, which proposes that DA, following release, diffuses in the extracellular space to reach its targets (Cragg and Rice 2004; Robinson et al. 2008; Rice et al. 2011). The efficacy of DA transmission would thus depend on its concentration, diffusion efficacy and on the affinity of its receptors (Cragg and Rice 2004). Because the DA D2 receptor (D2R) has a higher affinity than DA D1 receptor (D1R) (Tritsch and Sabatini 2012), it is thought that volume transmission has a smaller sphere of influence on the D1Rs (Rice et al. 2011). Our observation of a reduced distance between DA sources and their targets might indicate that following cocaine administration, the capacity of DA to also activate the D1Rs becomes facilitated.

Moss and Bolam termed the spatial distribution of DA boutons in the striatum a 'lattice', as it would correspond to a dense network of DA innervations through which volume transmission can act on all potential receptors (Moss and Bolam 2008; Sesack and Grace 2010). Therefore, our results show that this lattice becomes denser after cocaine administration, which could imply an enhanced effect of DA transmission. Our previous demonstration of increased glutamatergic synapse density after repeated cocaine administrations, albeit without an increase in the density of glutamatergic boutons (Heck et al. 2015), along with the present observation of increased DA bouton density and association with dendritic spines, shows that chronic cocaine administration modifies glutamatergic and DA connectivity in the ventral striatum. Such connectivity 
changes would likely contribute to synaptic plasticity and cellular adaptations induced by cocaine in striatal neurons.

Acknowledgements We thank Francois Tronche for providing the DAT-Cre mouse line, and the Cellular Imaging facility of the IBPS (Institut de Biologie Paris-Seine) for expert assistance on microscopy and metrological control of the setup. This work was supported by Centre National pour la Recherche Scientifique (CNRS), Institut National de la Santé et de la Recherche Médicale (INSERM), University Pierre and Marie Curie (UPMC), Agence Nationale de la Recherche (ANR), Fondation pour la Recherche Médicale (FRM) and the Labex Bio-Psy cluster of excellence. M.D.S. was a recipient of a fellowship from French Ministry of Research and Labex Bio-Psy. E.N.C. was supported by the Ecole de Neuroscience de Paris (ENP) and FRM.

\section{Compliance with ethical standards}

Conflict of interest The authors report no biomedical financial interests or potential conflicts of interest.

\section{References}

Addy NA, Daberkow DP, Ford JN, Garris PA, Wightman RM (2010) Sensitization of rapid dopamine signaling in the nucleus accumbens core and shell after repeated cocaine in rats. J Neurophysiol 104:922-931

Aragona BJ, Cleaveland NA, Stuber GD, Day JJ, Carelli RM, Wightman RM (2008) Preferential enhancement of dopamine transmission within the nucleus accumbens shell by cocaine is attributable to a direct increase in phasic dopamine release events. J Neurosci 28:8821-8831

Aransay A, Rodríguez-López C, García-Amado M, Clascá F, Prensa L (2015) Long-range projection neurons of the mouse ventral tegmental area: a single-cell axon tracing analysis. Front Neuroanat 9:59

Beitner-Johnson D, Nestler EJ (1991) Morphine and cocaine exert common chronic actions on tyrosine hydroxylase in dopaminergic brain reward regions. J Neurochem 57:344-347

Bernardinelli Y, Nikonenko I, Muller D (2014) Structural plasticity: mechanisms and contribution to developmental psychiatric disorders. Front Neuroanat 8:123

Bérubé-Carrière N, Guay G, Fortin GM, Kullander K, Olson L, Wallén-Mackenzie A, Trudeau LE, Descarries L (2012) Ultrastructural characterization of the mesostriatal dopamine innervation in mice, including two mouse lines of conditional VGLUT2 knockout in dopamine neurons. Eur J Neurosci 35:527-538

Chefer VI, Shippenberg TS (2002) Changes in basal and cocaineevoked extracellular dopamine uptake and release in the rat nucleus accumbens during early abstinence from cocaine: quantitative determination under transient conditions. Neuroscience 112:907-919

Cragg SJ, Rice ME (2004) DAncing past the DAT at a DA synapse. Trends Neurosci 27:270-277

De Mei C, Ramos M, Itaka C, Borrelli E (2009) Getting specialized: presynaptic and postsynaptic dopamine D2 receptors. Curr Opin Pharmacol 9:53-58

Descarries L, Watkins KC, Garcia S, Bosler O, Doucet G (1996) Dual character, asynaptic and synaptic, of the dopamine innervation in adult rat neostriatum: a quantitative autoradiographic and immunocytochemical analysis. J Comp Neurol 375:167-186
Di Chiara G, Imperato A (1988) Drugs abused by humans preferentially increase synaptic dopamine concentrations in the mesolimbic system of freely moving rats. Proc Natl Acad Sci USA 85:5274-5278

Gan WB, Grutzendler J, Wong WT, Wong RO, Lichtman JW (2000) Multicolor "DiOlistic" labeling of the nervous system using lipophilic dye combinations. Neuron 27:219-225

Gerfen CR, Surmeier DJ (2011) Modulation of striatal projection systems by dopamine. Annu Rev Neurosci 34:441-466

Gilles JF, Dos Santos M, Boudier T, Bolte S, Heck N (2017) DiAna, an ImageJ tool for object-based $3 \mathrm{D}$ co-localization and distance analysis. Methods 115:55-64

Heck N, Betuing S, Vanhoutte P, Caboche J (2012) A deconvolution method to improve automated 3D-analysis of dendritic spines: application to a mouse model of Huntington's disease. Brain Struct Funct 217:421-434

Heck N, Dos Santos M, Amairi B, Salery M, Besnard A, Herzog E, Boudier T, Vanhoutte P, Caboche J (2015) A new automated 3D detection of synaptic contacts reveals the formation of corticostriatal synapses upon cocaine treatment in vivo. Brain Struct Funct 220:2953-2966

Hübener M, Bonhoeffer T (2010) Searching for engrams. Neuron 67:363-371

Hyman SE, Malenka RC, Nestler EJ (2006) Neural mechanisms of addiction: the role of reward-related learning and memory. Annu Rev Neurosci 29:565-598

Ibáñez-Sandoval O, Tecuapetla F, Unal B, Shah F, Koós T, Tepper JM (2010) Electrophysiological and morphological characteristics and synaptic connectivity of tyrosine hydroxylase-expressing neurons in adult mouse striatum. J Neurosci 30:6999-7016

Johnson CM, Peckler H, Tai LH, Wilbrecht L (2016) Rule learning enhances structural plasticity of long-range axons in frontal cortex. Nat Commun 7:10785

Kalivas PW, Stewart J (1991) Dopamine transmission in the initiation and expression of drug- and stress-induced sensitization of motor activity. Brain Res Rev 16:223-244

Kuhar MJ, Pilotte NS (1996) Neurochemical changes in cocaine withdrawal. Trends Pharmacol Sci 17:260-264

Lammel S, Ion DI, Roeper J, Malenka RC (2011) Projection-specific modulation of dopamine neuron synapses by aversive and rewarding stimuli. Neuron 70:855-862

Lammel S, Lim BK, Malenka RC (2014) Reward and aversion in a heterogeneous midbrain dopamine system. Neuropharmacology 76 Pt B:351-359

Lee J, Parish CL, Tomas D, Horne MK (2011) Chronic cocaine administration reduces striatal dopamine terminal density and striatal dopamine release which leads to drug-seeking behaviour. Neuroscience 174:143-150

Lüscher C, Malenka RC (2011) Drug-evoked synaptic plasticity in addiction: from molecular changes to circuit remodeling. Neuron 69:650-663

Madisen L, Zwingman TA, Sunkin SM, Oh SW, Zariwala HA, Gu H, Ng LL, Palmiter RD, Hawrylycz MJ, Jones AR, Lein ES, Zeng $\mathrm{H}$ (2010) A robust and high-throughput Cre reporting and characterization system for the whole mouse brain. Nat Neurosci 13:133-140

Matsuda W, Furuta T, Nakamura KC, Hioki H, Fujiyama F, Arai R, Kaneko T (2009) Single nigrostriatal dopaminergic neurons form widely spread and highly dense axonal arborizations in the neostriatum. J Neurosci 29:444-453

Mejias-Aponte CA, Ye C, Bonci A, Kiyatkin EA, Morales M (2015) A subpopulation of neurochemically-identified ventral tegmental area dopamine neurons is excited by intravenous cocaine. J Neurosci 35:1965-1978 
Moss J, Bolam JP (2008) A dopaminergic axon lattice in the striatum and its relationship with cortical and thalamic terminals. J Neurosci 28:11221-11230

Pascoli V, Cahill E, Bellivier F, Caboche J, Vanhoutte P (2014) Extracellular signal-regulated protein kinases 1 and 2 activation by addictive drugs: a signal toward pathological adaptation. Biol Psychiatry 76:917-926

Pereira DB, Schmitz Y, Mészáros J, Merchant P, Hu G, Li S, Henke A, Lizardi-Ortiz JE, Karpowicz RJ Jr, Morgenstern TJ, Sonders MS, Kanter E, Rodriguez PC, Mosharov EV, Sames D, Sulzer D (2016) Fluorescent false neurotransmitter reveals functionally silent dopamine vesicle clusters in the striatum. Nat Neurosci 19:578-586

Rice ME, Patel JC, Cragg SJ (2011) Dopamine release in the basal ganglia. Neuroscience 198:112-137

Robinson DL, Hermans A, Seipel AT, Wightman RM (2008) Monitoring rapid chemical communication in the brain. Chem Rev 108:2554-2584

Robison AJ, Nestler EJ (2011) Transcriptional and epigenetic mechanisms of addiction. Nat Rev Neurosci 12:623-637

Robison AJ, Vialou V, Mazei-Robison M, Feng J, Kourrich S, Collins M, Wee S, Koob G, Turecki G, Neve R, Thomas M, Nestler EJ (2013) Behavioral and structural responses to chronic cocaine require a feedforward loop involving $\Delta$ FosB and calcium/calmodulin-dependent protein kinase II in the nucleus accumbens shell. J Neurosci 33:4295-4307

Rodriguez A, Ehlenberger DB, Dickstein DL, Hof PR, Wearne SL (2008) Automated three-dimensional detection and shape classification of dendritic spines from fluorescence microscopy images. PLoS One 3:e1997

Rodríguez-López C, Clascá F, Prensa L (2017) The mesoaccumbens pathway: a retrograde labeling and single-cell axon tracing analysis in the mouse. Front Neuroanat 11:25

Russo SJ, Dietz DM, Dumitriu D, Morrison JH, Malenka RC, Nestler EJ (2010) The addicted synapse: mechanisms of synaptic and structural plasticity in nucleus accumbens. Trends Neurosci 33:267-276

Schoonover CE, Tapia JC, Schilling VC, Wimmer V, Blazeski R, Zhang W, Mason CA, Bruno RM (2014) Comparative strength and dendritic organization of halamocortical and corticocortical synapses onto excitatory layer 4 neurons. J Neurosci 34:6746-6758

Schulz W (2002) Getting formal with dopamine and reward. Neuron $36: 241-263$

Sesack SR, Grace AA (2010) Cortico-basal ganglia reward network: microcircuitry. Neuropsychopharmacology 35:27-47
Sesack SR, Aoki C, Pickel VM (1994) Ultrastructural localization of D2 receptor-like immunoreactivity in midbrain dopamine neurons and their striatal targets. J Neurosci 14:88-106

Sorg BA, Chen SY, Kalivas PW (1993) Time course of tyrosine hydroxylase expression after behavioral sensitization to cocaine. J Pharmacol Exp Ther 266:424-430

Steketee JD, Kalivas PW (2011) Drug wanting: behavioral sensitization and relapse to drug-seeking behavior. Pharmacol Rev 63:348-365

Thomas MJ, Beurrier C, Bonci A, Malenka RC (2001) Long-term depression in the nucleus accumbens: a neural correlate of behavioral sensitization to cocaine. Nat Neurosci 4:1217-1223

Todtenkopf MS, Stellar JR (2000) Assessment of tyrosine hydroxylase immunoreactive innervation in five subregions of the nucleus accumbens shell in rats treated with repeated cocaine. Synapse 38:261-270

Todtenkopf MS, De Leon KR, Stellar JR (2000) Repeated cocaine treatment alters tyrosine hydroxylase in the rat nucleus accumbens. Brain Res Bull 52:407-411

Tritsch NX, Sabatini BL (2012) Dopaminergic modulation of synaptic transmission in cortex and striatum. Neuron 76:33-50

Turiault M, Parnaudeau S, Milet A, Parlato R, Rouzeau JD, Lazar M, Tronche F (2007) Analysis of dopamine transporter gene expression pattern-generation of DAT-iCre transgenic mice. FEBS J 274:3568-3577

Uchigashima M, Ohtsuka T, Kobayashi K, Watanabe M (2016) Dopamine synapse is a neuroligin-2-mediated contact between dopaminergic presynaptic and GABAergic postsynaptic structures. Proc Natl Acad Sci USA 113:4206-4211

Wierenga CJ, Becker N, Bonhoeffer T (2008) GABAergic synapses are formed without the involvement of dendritic protrusions. Nat Neurosci 11:1044-1052

Xu C, Zhao J, Liu Y, Zeng X, Jia Y, Wang Y, Jiang X, Xu Q (2012) Dopaminergic axons preferentially innervate dendritic spines with hyperactive glutamatergic synapses in the rat striatum. Brain Res 1486:92-102

Yang Y, Liu DQ, Huang W, Deng J, Sun Y, Zuo Y, Poo MM (2016) Selective synaptic remodeling of amygdalocortical connections associated with fear memory. Nat Neurosci 19:1348-1355

Yung KK, Bolam JP, Smith AD, Hersch SM, Ciliax BJ, Levey AI (1995) Immunocytochemical localization of D1 and D2 dopamine receptors in the basal ganglia of the rat: light and electron microscopy. Neuroscience 65:709-730 